\title{
Urban Noise and Surrounding City Morphology Influence Green Space Occupancy by Native Birds in a Mediterranean-type South American Metropolis
}

\section{Constanza Arévalo}

Pontificia Universidad Católica de Chile

Juan David Amaya-Espinel

Pontificia Universidad Javeriana

Cristian Henríquez

Pontificia Universidad Católica de Chile

José Tomás Ibarra

Pontificia Universidad Católica de Chile

Cristián Bonacic ( $\square$ bona@uc.cl)

Pontificia Universidad Católica de Chile

\section{Research Article}

Keywords: Urban avoiders, urban utilizers, urban dwellers, anthrophony, soundscape, urban morphology, urban sprawl

Posted Date: January 10th, 2022

DOI: https://doi.org/10.21203/rs.3.rs-1217978/v1

License: (c) (i) This work is licensed under a Creative Commons Attribution 4.0 International License.

Read Full License

Version of Record: A version of this preprint was published at Scientific Reports on March 16th, 2022. See the published version at https://doi.org/10.1038/s41598-022-08654-7. 


\section{Abstract}

Urban green spaces provide natural habitat for birds in urban landscapes, yet the effects of noise and surrounding urban morphology on bird community structure and distribution are not well understood in Latin America, the second most urbanized region in the world. Santiago of Chile is the single city belonging to the Mediterranean ecosystem in South America and is subject to extensive urbanization as seen throughout Latin America. We examined the role of 65 urban green spaces ( 6 large: PAR and 59 small: SGS) in harboring native birds during winter 2019, analyzing the quality of green areas in terms of vegetation (i.e. NDVI, native vegetation, and tree cover), exotic bird species, noise levels, and surrounding urban matrix (i.e. building height and cover). Significantly higher noise levels were detected in SGS, along with significantly greater exotic bird $(n=4)$ richness and abundance than PAR, which possessed significantly greater native bird $(n=25)$ richness and abundance. Native birds were more abundant than exotic birds in green spaces with average noise levels $<52 \mathrm{~dB}$ and average NDVI $>0.5$. Occupancy models indicate that green space occupancy by $50 \%$ of modeled native bird species was influenced by maximum noise levels, playing a larger role than vegetation (30\%) and the urban matrix $(0 \%)$. We stress the importance of developing networks of large green spaces in rapidly urbanizing regions, with abundant tree cover, surrounded by smaller urban morphology, and regulating noise levels to ensure the conservation of native bird communities in cities, particularly those that are threatened.

\section{Introduction}

Over half (55\%) of the world's population resides in urban areas, a number expected to reach $68 \%$ by $2050^{[35]}$. Urban population growth has been particularly rapid in Latin America and the Caribbean, making up the world's second most urbanized region with $81 \%$ of its population living in urban areas, and an expected $90 \%$ by $2050^{[35]}$. Urbanization poses a risk to biodiversity with the loss and fragmentation of natural habitats for countless species around the globe, while a rise in noise, light, and chemical pollution associated with urbanization further impact urban species by influencing physiological and behavioral changes $[18,34]$, resulting in serious challenges for biodiversity conservation.

Species can be categorized according to their tolerance to urbanization, classifying them based on their responses to urban development $[5,10,14,22]$. Three categories of urbanization tolerance represent a response gradient: urban avoiders range from extirpated in developed areas to self-sustaining in networks of natural areas within cities, urban utilizers range from occasionally using urban resources to breeding in developed areas, and urban dwellers range from having viable populations in both natural and urban areas to being dependent on urban areas for their survival ${ }^{[10]}$.

As the global population becomes increasingly more urban, we can expect a rise in anthropogenic noise within the world's soundscapes. Sound serves as an important stimulus for numerous species, carrying large amounts of information used in intra- and inter-species communication [21]. When noise becomes permanent in a system, as occurs with urbanization, communication among individuals becomes limited, altering their ability to forage, hide from predators, and reproduce $[20,27,37]$. 
Certain animal species, such as songbirds, are particularly susceptible to changes in the soundscape ${ }^{[27,}$ ${ }^{30}$, with studies attributing reduced reproductive success, bird fitness, bird densities, bird community richness and diversity, as well as changes to species interactions in urban areas to anthropogenic noise $[12,27,32]$. Birds may be excluded on a purely acoustic basis from otherwise suitable habitats ${ }^{[30,36]}$, with the anthrophony (human-produced sounds) acting as a source of habitat degradation and fragmentation that affects the distribution of bird communities. This acoustic fragmentation, responsible for developing song differences between urban and non-urban birds of the same species $[6,23,24,26,33]$, may result in reproductive isolation ${ }^{[25]}$.

Amid the negative impacts of urbanization on bird communities, urban green spaces provide pockets of habitat for birds. Studies conducted in urban parks have found that bird species richness is positively related to the area of green spaces and negatively related to anthropogenic noise levels ${ }^{[15,16,28]}$. As residential density increases with a growing global population, urban parks are predicted to lose their capacity to provide habitat for urban wildlife ${ }^{[16]}$. A series of ecological conditions that occur in city green spaces influence bird richness and abundance. First, road coverage and building density. Second, the presence of invasive species and urban-dweller generalist bird species, and third, anthropogenic noise levels ${ }^{[1]}$. Particularly, the effects of anthropogenic noise on bird communities have been suggested to go beyond the community level, altering bird species richness and composition at a regional scale ${ }^{[15]}$.

Due to the significance of urban green spaces for the survival and reproduction of bird communities within cities, it is imperative that the often-unnoticed effects of urban morphology surrounding these sites and the noise within them be studied to better understand how they affect bird community structure and distribution. South America, as one of the fastest urbanizing regions of the world, is lacking in such studies, with most research on wildlife and anthropogenic noise being conducted in North America and Europe ${ }^{[19]}$. Santiago of Chile is one of the large metropolises of Latin America and stands out for its high-density value in terms of inhabitants per hectare, along with cities like Bogotá, Caracas, São Paulo, and Mexico City ${ }^{[8]}$. With $88.4 \%$ of its population currently residing in urban areas ${ }^{[17]}$, Chile is one of the South American countries with the highest urban population concentrated in the single Mediterraneantype ecosystem of South America. Even though birds are the taxa with the least number of endemic species relative to other terrestrial vertebrates in Chile, they include unique species like the Chilean mockingbird (Mimus thenca), moustached turca (Pteroptochos megapodius), slender-billed parakeet (Enicognathus leptorhynchus), among others whose conservation status has been affected by intensive agriculture and urbanization.

This study aimed to understand the influence of urbanization on native bird species richness, abundance, and green space occupancy in an exemplary South American metropolis. Specifically, we sought to determine how native bird richness and abundance in urban green spaces are influenced by anthropogenic noise, the surrounding city matrix, exotic bird species, and vegetation in green spaces. We further used occupancy modeling techniques to predict native urban avoider, urban utilizer, and urban dweller bird species presence in urban green spaces, given noise levels, surrounding building height and 
cover, and vegetation variables. We expected: i) native bird richness and abundance to decrease with rising noise levels, building height, building cover, and exotic bird abundance; ii) probability of green space occupancy by native birds to decrease as noise levels, building height, and building cover increase; and iii) native urban avoider species to show the most sensitivity to noise and urban morphology, and thus show increased occupancy in large urban parks, and native urban dwellers to be the most tolerant to urbanization and be more commonly found in small green spaces.

\section{Results}

We detected 29 bird species (Appendix S5) ( $86 \%$ native [ $n=25]$ to Chile; $14 \%$ exotic [ $n=4]$ ) within the green spaces of Santiago, all categorized as being of 'least concern' in the IUCN Red List. Generally, native bird richness and abundance were greatest in large urban parks (PAR; area > 10 ha), while exotic bird abundance was greater in small green spaces (SGS; area < 10 ha) (Appendix S2). Native bird richness and abundance decreased with increasing noise levels and building height surrounding green spaces, while increasing with vegetation cover (Appendix S3). Exotic bird abundance increased with rising noise levels and building height, decreased with vegetation cover, and exotic bird richness decreased with tree cover (Appendix S3). Native birds reached lesser abundances than exotic birds when average noise levels reached over $52 \mathrm{~dB}$, and, once vegetation cover in green spaces reached an average NDVI value of 0.5 or more, native birds tended to reach higher abundances than exotic birds (Appendix S4).

\section{Native bird tolerances to urbanization conditions}

Average noise levels in green spaces shared significant correlations with the richness and abundance of native urban avoider $(n=7)$, urban utilizer $(n=8)$, and urban dweller $(n=12)$ species, indicating an important negative trend in the richness and abundance of all native bird species as average noise levels rose in green spaces (Table 1). Additionally, a significant positive correlation was found between NDVI and urban utilizer abundance in green spaces, a negative relation between native vegetation in green spaces and native urban utilizer richness and abundance, as well as a significant negative relation between average building height surrounding green spaces and urban avoider and urban dweller species richness and abundance. Native urban avoider species displayed the least tolerance to anthropogenic noise and building height surrounding green spaces, rarely detected in green spaces with average noise levels over $60 \mathrm{~dB}$ and average building height over $10 \mathrm{~m}$, with most found in green spaces with average noise levels of 50 - $55 \mathrm{~dB}$ and surrounded by buildings $4-6 \mathrm{~m}$ tall (Fig. 1a). Unlike most native urban utilizer species, the green-backed firecrown (Sephanoides sephaniodes) was found in most green spaces, for which reason it was considered separately from other urban utilizers (Fig. 1b). The green backed firecrown's tolerance to anthropogenic noise was more like that of urban dwellers, found throughout most noise levels, though more commonly in green spaces with average noise levels between 50 and $65 \mathrm{~dB}$ (Fig. 1C), while other urban utilizers were most detected in green spaces with average noise levels around $55 \mathrm{~dB}$, decreasing rapidly with noise levels higher than that (Fig. 1b). All native birds showed sensitivity to building height, with few native birds detected in green spaces surrounded by buildings more than $10 \mathrm{~m}$ 
tall on average. Meanwhile, there was not sufficient evidence that building cover surrounding green spaces, nor tree cover in green spaces, share significant relations with native urban avoider, urban utilizer, and urban dweller richness and abundance in green spaces (Table 1).

Generally, noise levels were found to be greatest in green spaces surrounded by taller urban morphology, while there was not enough evidence indicating that building cover shared a relation with noise.

Table 1

Pearson/Spearman correlation coefficients and associated p-values for native urban avoider, urban utilizer, and urban dweller groups in green spaces of Santiago, Chile.

\begin{tabular}{|c|c|c|c|c|c|c|}
\hline & \multicolumn{3}{|l|}{ Richness } & \multicolumn{3}{|l|}{ Abundance } \\
\hline & $\begin{array}{l}\text { Urban } \\
\text { avoiders }\end{array}$ & $\begin{array}{l}\text { Urban } \\
\text { utilizers }\end{array}$ & $\begin{array}{l}\text { Urban } \\
\text { dwellers }\end{array}$ & $\begin{array}{l}\text { Urban } \\
\text { avoiders }\end{array}$ & $\begin{array}{l}\text { Urban } \\
\text { utilizers }\end{array}$ & $\begin{array}{l}\text { Urban } \\
\text { dwellers }\end{array}$ \\
\hline \multirow[t]{2}{*}{ Average noise } & -0.37 & -0.29 & -0.24 & -0.35 & -0.31 & -0.36 \\
\hline & $p=0.002^{*}$ & $\begin{array}{l}p= \\
0.016^{*}\end{array}$ & $p=0.049 *$ & $p=0.003^{*}$ & $\begin{array}{l}p= \\
0.009 *\end{array}$ & $p=0.003^{*}$ \\
\hline \multirow[t]{2}{*}{ NDVI } & -0.10 & 0.17 & 0.01 & -0.07 & 0.38 & -0.03 \\
\hline & $p=0.399$ & $p=0.172$ & $p=0.904$ & $p=0.568$ & $\begin{array}{l}\mathrm{p}= \\
0.001 *\end{array}$ & $p=0.783$ \\
\hline \multirow[t]{2}{*}{ Tree cover } & -0.06 & -0.14 & 0.02 & -0.02 & 0.08 & -0.09 \\
\hline & $p=0.601$ & $p=0.256$ & $p=0.861$ & $p=0.860$ & $p=0.530$ & $p=0.483$ \\
\hline \multirow[t]{2}{*}{ Native vegetation } & -0.05 & -0.25 & -0.01 & -0.03 & -0.27 & -0.13 \\
\hline & $p=0.695$ & $\begin{array}{l}\mathrm{p}= \\
0.041 *\end{array}$ & $p=0.904$ & $p=0.834$ & $\begin{array}{l}\mathrm{p}= \\
0.028^{*}\end{array}$ & $p=0.270$ \\
\hline \multirow{2}{*}{$\begin{array}{l}\text { Average building } \\
\text { height }\end{array}$} & -0.25 & 0.02 & -0.24 & -0.25 & -0.20 & -0.35 \\
\hline & $p=0.040 *$ & $p=0.863$ & $p=0.049 *$ & $p=0.036^{*}$ & $p=0.095$ & $p=0.003^{*}$ \\
\hline \multirow{2}{*}{$\begin{array}{l}\text { Proportion } \\
\text { building cover }\end{array}$} & 0.02 & -0.18 & 0.02 & 0.01 & -0.09 & -0.05 \\
\hline & $p=0.872$ & $p=0.149$ & $p=0.873$ & $p=0.954$ & $p=0.460$ & $p=0.675$ \\
\hline
\end{tabular}

\section{Detection and occupancy probability models}

Detectability remained constant across surveys and surveyed sites for the Chilean mockingbird (Mimus thenca), the only endemic bird species detected in the study area, not affected by any one variable. Detectability varied across survey sites for the remaining 13 native bird species, changing with the maximum noise level recorded during surveys (14\%), vegetation cover (29\%), tree cover (21\%), native vegetation (14\%), building cover surrounding green spaces (7\%), and with a combination of average 
building height and maximum noise level (7\%). Detectability decreased with rising noise levels, native vegetation, building height, and building cover surrounding green spaces, while increasing with vegetation cover.

Green space occupancy was most influenced by the maximum noise levels recorded in green spaces for $50 \%$ of modeled native bird species (Table 2), with urban avoider and urban utilizer species showing the lowest noise tolerances, and exotic species displaying the highest tolerances to noise, with some species present especially in high-noise green spaces (the rock pigeon (Columba livia) and the monk parakeet (Myiopsitta monachus)). The picui ground dove (Columbina picui) and long-tailed meadowlark (Leistes loyca) were the modeled native species most sensitive to noise, their occupancy probabilities decreasing rapidly as the average maximum noise level of a green space reached $55-65 \mathrm{~dB}$, approaching an occupancy probability of 0 when average maximum noise levels reached over $65 \mathrm{~dB}$ (Fig. 2a \& b). The common diuca finch (Diuca diuca) and rufous-collared sparrow (Zonotrichia capensis) were also quite sensitive to noise, their occupancy probabilities decreasing as average maximum noise levels reached above $55 \mathrm{~dB}$ (Fig. 2b \& c), but the decrease in their occupancy probabilities was more gradual than for the other two species. The austral thrush (Turdus falcklandii) displayed the least sensitivity to noise, present in all green spaces with an average maximum noise level below approximately $73 \mathrm{~dB}$ (Fig. 2c). Following maximum noise level as the most influential covariate in occupancy probability, tree cover in green spaces was most influential for $20 \%$ of native birds, green space occupancy remained constant across sites for $20 \%$ of native birds, and, for the remaining $10 \%$, occupancy was influenced by vegetation cover (Table 2). 
Table 2

Best occupancy detection probability models for bird species detected in Santiago, Chile, showing estimated detection (p) and occupancy $(\Psi)$ probabilities.

\begin{tabular}{|c|c|c|c|c|c|c|}
\hline $\begin{array}{l}\text { Urbanization } \\
\text { tolerance }\end{array}$ & Common name $\mathrm{a}^{\mathrm{a}}$ & Scientific name & Model $^{b}$ & Est. $p$ & $\begin{array}{l}\text { Naïve } \\
\Psi\end{array}$ & $\begin{array}{l}\text { Est. } \\
\Psi\end{array}$ \\
\hline Avoider & $\begin{array}{l}\text { Long-tailed } \\
\text { meadowlark }\end{array}$ & Leistes loyca & $\begin{array}{l}\Psi(\mathrm{MNA}) \\
\mathrm{p}(\mathrm{NDVI})\end{array}$ & 0.565 & 0.073 & 0.048 \\
\hline Utilizer & $\begin{array}{l}\text { Picui ground } \\
\text { dove }\end{array}$ & Columbina picui & $\begin{array}{l}\psi(\mathrm{MNA}) \\
\mathrm{p}(\mathrm{NV})\end{array}$ & 0.105 & 0.130 & 0.199 \\
\hline Utilizer & $\begin{array}{l}\text { Common diuca } \\
\text { finch }\end{array}$ & Diuca diuca & $\begin{array}{l}\Psi(\text { MNA }) \\
\text { p(Tree) }\end{array}$ & 0.249 & 0.203 & 0.374 \\
\hline Utilizer & $\begin{array}{l}\text { Chilean } \\
\text { mockingbird }\end{array}$ & Mimus thenca & $\psi(\mathrm{NDVI}) \mathrm{p}()$. & 0.456 & 0.275 & 0.327 \\
\hline Utilizer & $\begin{array}{l}\text { Green-backed } \\
\text { firecrown }\end{array}$ & $\begin{array}{l}\text { Sephanoides } \\
\text { sephaniodes }\end{array}$ & $\begin{array}{l}\Psi(\text { Tree }) \\
\text { p(NDVI) }\end{array}$ & 0.909 & 0.913 & 0.970 \\
\hline Utilizer & $\begin{array}{l}\text { Black-chinned } \\
\text { siskin }\end{array}$ & Spinus barbatus & $\psi(.) \mathrm{p}(\mathrm{MN})$ & 0.116 & 0.072 & 0.218 \\
\hline Utilizer & $\begin{array}{l}\text { Southern } \\
\text { lapwing }\end{array}$ & Vanellus chilensis & $\begin{array}{l}\psi(\text { Tree }) \\
\mathrm{p}(\mathrm{NDVI})\end{array}$ & 0.196 & 0.145 & 0.281 \\
\hline Urban dweller & Feral Pigeon* & Columba livia & $\begin{array}{l}\Psi(\mathrm{MNA}+\mathrm{H}) \\
\mathrm{p}(\mathrm{NDVI})\end{array}$ & 0.933 & 0.884 & 0.952 \\
\hline Urban dweller & $\begin{array}{l}\text { Chimango } \\
\text { caracara }\end{array}$ & $\begin{array}{l}\text { Milvago } \\
\text { chimango }\end{array}$ & $\psi(.) \mathrm{p}(\mathrm{MN})$ & 0.420 & 0.507 & 0.658 \\
\hline Urban dweller & Shiny cowbird* & $\begin{array}{l}\text { Molothrus } \\
\text { bonariensis }\end{array}$ & $\begin{array}{l}\psi(\text { MNA) } \\
\text { p(Tree) }\end{array}$ & 0.457 & 0.652 & 0.883 \\
\hline Urban dweller & Monk parakeet* & $\begin{array}{l}\text { Myiopsitta } \\
\text { monachus }\end{array}$ & $\Psi(\mathrm{NV}) \mathrm{p}(\mathrm{D})$ & 0.728 & 0.812 & 0.879 \\
\hline Urban dweller & House sparrow* & $\begin{array}{l}\text { Passer } \\
\text { domesticus }\end{array}$ & $\begin{array}{l}\psi(N D V I) \\
p(N D V I)\end{array}$ & 0.439 & 0.449 & 0.482 \\
\hline Urban dweller & Austral thrush & Turdus falcklandii & $\psi(\mathrm{MNA}) \mathrm{p}(\mathrm{D})$ & 0.991 & 0.986 & 0.999 \\
\hline Urban dweller & $\begin{array}{l}\text { Rufous-collared } \\
\text { sparrow }\end{array}$ & $\begin{array}{l}\text { Zonotrichia } \\
\text { capensis }\end{array}$ & $\begin{array}{l}\psi(\mathrm{MNA}) \\
\mathrm{p}(\mathrm{NV})\end{array}$ & 0.621 & 0.797 & 0.895 \\
\hline \multicolumn{7}{|c|}{${ }^{a}$ Exotic species marked with * } \\
\hline \multicolumn{7}{|c|}{$\begin{array}{l}\text { b Variables: maximum noise level (MN), average maximum noise level (MNA), vegetation cover } \\
\text { (NDVI), proportion native vegetation (NV), proportion tree cover (Tree), average building height } \\
\text { surrounding green space (H), building cover surrounding green space (D), constant occupancy across } \\
\text { sites (.) }\end{array}$} \\
\hline
\end{tabular}




\section{Discussion}

Our research determined noise to share an important negative relationship with native bird richness and abundance and to be the most limiting factor in green space occupancy by native bird species, more so than the type and amount of vegetation present in urban green spaces, and more so than urbanization itself, represented as building height and cover surrounding green spaces. Thus, noise acts as an invisible source of habitat degradation, limiting the bird species capable of inhabiting an area, regardless of whether the appropriate vegetative conditions exist.

As predicted, native urban avoiders reached their maximum abundances in PAR, which, given their high vegetation cover and large size, act as patches of natural habitat in cities. Native urban utilizers tended to be found in more suburban areas, and urban dwellers, both native and exotic, were detected in green spaces of all noise levels. All exotic bird species were urban dwellers, referring to their high tolerance to urbanization ${ }^{[1,10]}$, thus reaching the high abundances observed, particularly in SGS.

SGS possessed higher average noise levels and greater exotic bird abundance than PAR, which presented significantly higher numbers of native bird richness and abundance. The influence of noise on native bird species first becomes evident when we consider that native bird abundance tended to rise above the generally high abundance of exotic birds when average noise levels in green spaces reached below $52 \mathrm{~dB}$ (it should be noted that, according to the Chilean Noise Norm $N^{\circ} 146$, the maximum allowable noise levels generated by fixed sources in residential areas of Santiago is $55 \mathrm{~dB}$ during the day, 7 a.m. -9 p.m.). The negative relations between noise and urban avoider, urban utilizer, and urban dweller species richness and abundance further indicate how noise may be regulating the native bird species present in green spaces, affecting urban avoider richness the most and urban dweller richness the least, while influencing the abundance of all native bird species rather similarly. Meanwhile, building height surrounding green spaces negatively influenced native urban avoider and urban dweller richness and abundance, with the greatest influence on urban dweller abundance, yet all native birds were less likely to be detected in green spaces surrounded by buildings over $10 \mathrm{~m}$ tall on average.

The importance of vegetation for native bird communities also cannot be denied, given that native birds reached higher abundances than exotic birds when vegetation cover reached an average NDVI value greater than 0.5. Results from this study thus suggest that exotic birds begin to replace native birds in terms of abundance as noise levels rise in urban green spaces, vegetation cover decreases, and building height surrounding green spaces increases, with native urban avoider species being the least tolerant to the influences of urbanization, and, consequently, the first to disappear when noise levels and building height become too great.

Detection probability models found native bird detectability to mostly increase with vegetation cover and tree cover in urban green spaces, except for the common diuca finch, whose detectability decreased with rising tree cover. Some of the bird species that displayed the lowest detection probabilities, such as the picui ground dove and fire-eyed diucon (Xolmis pyrope), are not frequently found in cities and possess 
vocalizations that are unlikely to be heard well in high-noise areas due to their low frequencies, making them more easily masked by the anthrophony, characterized by its low frequency and high intensity [13] Consequently, birds whose vocalizations are similar in frequency and amplitude to the anthrophony were more commonly or exclusively found in green spaces that registered low noise levels, their detectability also decreasing with rising noise, as was the case with the fire-eyed diucon.

Urban green space occupancy by native bird species was mainly influenced by average maximum noise levels recorded in green spaces. Of the modeled native species, the long-tailed meadowlark and the picui ground dove, an urban avoider and an urban utilizer species respectively, were the species most sensitive to noise, their probability of occupying green spaces with average maximum noise levels over $55 \mathrm{~dB}$ decreasing rapidly and approaching zero when over $65 \mathrm{~dB}$. Meanwhile, the austral thrush, an urban dweller species, was by far the most tolerant to noise of the native birds, its presence probability just beginning to decrease when average maximum noise levels reached over $73 \mathrm{~dB}$ in green spaces. The differing tendencies of urban avoiders, urban utilizers, and urban dwellers to occupy green spaces of varying noise levels is thus evident, with native urban dweller species more likely to occupy higher noise urban green spaces than urban avoiders and utilizers, seemingly more adapted to the high noise levels that come with inhabiting a busy city. Nonetheless, although native urban dwellers displayed greater noise tolerances than urban avoiders and utilizers, their presence in city parks can also be expected to diminish if noise levels become too high, which for the most tolerant of the native birds, means reaching an average maximum level of $73 \mathrm{~dB}$ or more, but $55 \mathrm{~dB}$ or more for less tolerant species.

No relation was found between vegetation cover and noise, and some of the highest noise levels were recorded in PAR. This suggests that PAR, often considered to be quiet and peaceful areas to escape the busyness of city life, can reach noise levels as high as those recorded in SGS, reducing the quality of the greatest sources of natural habitat for birds and other wildlife in cities.

The results from this study regarding the influence of noise on bird communities support previous studies indicating that birds may be excluded from suitable habitats on account of the acoustic conditions of the local environment ${ }^{[30,36]}$. Despite abundant vegetation in PAR and some SGS, certain bird species, particularly urban avoiders and utilizers, were less likely to occupy areas that presented high noise levels. However, it is important to consider other potential influencing factors, such as predators (e.g., dogs and cats) and food availability, both of which could be linked to pedestrians and could therefore also increase noise levels in green spaces. Furthermore, in an effort to focus on the influence of anthropogenic variables on urban birds (i.e., urban morphology, noise, and vegetation type and cover), this study did not consider the size of urban green spaces as a variable in occupancy modeling, but as the results of this study and others have suggested, green space size is likely an influencing factor that should be considered in future studies.

Measures to control the COVID-19 pandemic have significantly reduced noise levels in major cities worldwide ${ }^{[2,4,9]}$. Noise reduction in the San Francisco Bay Area, characterized by a Mediterranean climate like Santiago, resulted in songbirds rapidly occupying newly available acoustic niches within 
urban soundscapes and maximizing communication through higher performance songs ${ }^{[9]}$. Consequently, native bird species not commonly found in high-noise areas, mainly urban avoider and utilizer species, may now be found in greater abundance at the community level in urban green spaces where they had been scarce or non-existent during this study, conducted pre-pandemic. Furthermore, if average noise levels dropped below $52 \mathrm{~dB}$ in Santiago green spaces due to region-wide shut-down measures, native birds may reach higher abundances than exotic birds. The negative effects of urban noise on bird communities are extensive, yet recent research indicating birds' rapid adaptability and improved vocal performance when noise levels are significantly lowered provides hope. Native bird species susceptible to noise may stand a chance despite growing urbanization, if noise levels in urban green spaces are regulated.

Rapid urban expansion in Latin America places natural ecosystems at great risk, reducing or altogether eliminating natural habitats for native birds and other wildlife, making urban green spaces necessary for their persistence, especially in biodiversity hotspots like central Chile. As this study illustrates, noise associated with urbanization plays a significant role in influencing green space occupancy by native bird species, and, quite possibly, other animal species dependent on acoustic signaling (e.g., amphibians and mammals). Given the recreational role of urban green spaces in cities, noise regulation within these areas should be considered, while also considering how city morphology may impact bird communities. This study exemplifies how, in addition to noise, the size of urban green spaces and the vegetation cover in them, particularly tree cover, are vital aspects to consider in city planning in order to preserve native bird communities in urban systems. Large urban parks held significantly richer bird communities than small green spaces, with greater native bird richness and abundance. Therefore, it is imperative that science and city planning collaborate to develop cities with networks of large green spaces with abundant tree cover, surrounded by smaller urban morphology, where noise is regulated and maintained at tolerable levels for native birds. There is a clear need to move towards biophilic city planning to harmonize urban growth and the protection and expansion of networks of green areas that generate habitat for birds that, in turn, provide important ecosystem services to cities.

\section{Methods}

\section{Bird surveys and noise measurements}

We conducted this study in 59 small green spaces (SGS) and 6 large urban parks (PAR) in the Metropolitan Region of Santiago (MRS) in central Chile (Appendix S1). Located between the Andes mountains and a coastal mountain range, the MRS possesses a semi-arid Mediterranean climate, with hot, dry summers and cold, wet winters ${ }^{[31]}$. Due to its highly endemic vegetation, the Mediterranean region of central Chile is considered a biodiversity hotspot, with sclerophyllous shrubland and woodland making up the dominant vegetation ${ }^{[3]}$. The region holds about $40 \%$ of Chile's population, of which $96.3 \%$ resides in urban areas ${ }^{[17]}$. SGS ranged in size from 0.5 to 2 ha and were independent of one another, separated by a distance of at least $250 \mathrm{~m}$ from other surveyed SGS and PAR ${ }^{[1]}$. We sampled 6 PAR of 
area $>10$ ha as control points, with 1-3 survey points in each, depending on park size, for a total of 10 PAR sample sites, ensuring a distance of at least $250 \mathrm{~m}$ between survey points. Green spaces were identified using a digital layer of green area developed for the MRS at a scale of 1:5000, updated using Landsat 8 OLI Thematic Mapper satellite imagery from November $2013^{[1]}$.

We conducted point count surveys at all SGS and PAR once a month during winter 2019 (May-August), for a total of three surveys per green space. To capture the morning chorus, the point of maximum biophony activity ${ }^{[29]}$, we conducted bird surveys within four hours from sunrise. We altered the order of bird surveys each month so as to never sample a green space at the same time, to account for varying noise levels throughout the morning, which in turn could alter the bird species present during a survey [7]. We used a radius of $50 \mathrm{~m}$, recording all bird species detected visually and acoustically during a 10-minute period, along with the number of individuals of each species. Additionally, we determined noise levels at the beginning and end of each bird survey for a period of 30 seconds using the NIOSH Sound Level Meter app for smartphones combined with the Dayton Audio iMM- 6 calibrated measurement microphone, measuring average continuous equivalent sound pressure levels $\left(\mathrm{LA}_{\mathrm{eq}}\right)$ and maximum equivalent sound pressure levels $\left(L_{\max }\right)$ using slow $A$ frequency weighting.

\section{Urban matrix measurements}

As a measure of the urban matrix resulting from urbanization, we considered average building height and building cover within a circular buffer of $200 \mathrm{~m}$ from the central point of each urban green space,

obtained in $2015^{[1]}$. We calculated the proportion of building cover within the buffer using the Normalized Difference Vegetation Index (NDVI) to map the percentage of the buffer covered by vegetation and took the difference. Average building height was calculated using a raster layer with cadastral information for the study area. Building height was validated through Google Street View 3D models, estimating a height of $2.70 \mathrm{~m}$ per built floor. We determined average building height surrounding urban green spaces for a random sample $(n=15)$ of SGS in 2020 using the same method, but no significant differences were found between 2015 and 2020 urban morphology data (t-test $p$-value $=0.922$ ).

\section{Vegetation cover and composition}

We determined the average NDVI for each urban green space as an estimate of vegetation cover, using QGIS 2.18.23 to process Landsat 8 OLI Thematic Mapper satellite imagery of the study area taken in September 2019, with values close to zero indicating bare soil or urban areas, and values closer to 1 implying dense vegetation.

Additionally, we estimated the proportion of native vegetation and tree cover (vegetation $>2 \mathrm{~m}$ tall) in each green space, following 40 points along the border of $50 \times 50 \mathrm{~m}$ plots at the center of each survey site (every $2.5 \mathrm{~m}$ along 4 transects of $50 \mathrm{~m}$ ), using frequency as a measure of cover ${ }^{[1]}$.

\section{Statistical analysis}


We considered richness to be the total number of bird species detected per urban green space during the study period and abundance to be the average number of individuals of each species detected per urban green space throughout the point counts. $\mathrm{LA}_{\mathrm{eq}}$ measurements were averaged to obtain the average noise level per green space (NW), and the same was done with $L_{\max }$ measurements, giving an average maximum noise level per green space (MNA). We tested all site variables (NW, MNA, average building height, building cover, NDVI, proportion native vegetation, and tree cover) for collinearity. In the event of high correlation $(r>0.7)$, only one variable was considered, as was the case with NW and MNA. Correlation analyses were also run between site variables and species richness and abundance.

We modeled native and exotic bird abundance in relation to site variables with generalized linear models (GLMs) using R version 3.6.0 and the R package ggplot2. To visualize the distribution of native birds under the influence of the most significant urban variables, we created scatterplots with marginal density plots for each urban tolerance category (urban avoiders, urban utilizers, urban dwellers) considering the urbanization variables that had the highest correlations with native bird richness and abundance. Species urban tolerances were determined following the classification method proposed by Fischer et al. (2015), considering the spatial and temporal frequency of species observations ${ }^{[1]}$.

\section{Detection and occupancy modeling}

We developed occupancy models using the $\mathrm{R}$ package unmarked ${ }^{[11]}$ to determine the true presence and absence probabilities of bird species at sampling sites. The probability of detecting a species and its probability of occupying a green space both follow a Bernoulli model to which we incorporated data from repeated surveys, as well as site (i.e. MNA, average building height surrounding green spaces $[\mathrm{H}]$, proportion of building cover surrounding green spaces [D], vegetation cover [NDVI], proportion of tree cover [Tree], and proportion of native vegetation in green spaces [NV]) and observation (i.e. maximum noise level during point count [MN]) covariates to test how these variables influence species detection and green space occupancy.

We determined the best detection probability model for each bird species detected, keeping occupancy $(\psi)$ constant across all sites and considering all observation and site covariates. Models were selected based on Akaike Information Criteria (AIC), where only models with AIC $\leq 2.0$ were considered in the model selection process. The model with the lowest AIC value and greatest Akaike weight $(w)$ was selected as the best model for each bird species.

The best detectability model for each bird species was then incorporated into the occupancy model selection process, where we tested all site covariates to obtain the best occupancy model. Model selection was carried out in the same manner as with detectability, using AIC to determine the best model for each bird species. Species that displayed estimated detection probabilities $(p)<0.1$ or that displayed a broad confidence interval associated with their occupancy probability $(\psi)$ were not included due to the uncertainty associated with the expected detection of those species and/or green space occupancy by those species. 15 native bird species, mainly urban avoiders and urban utilizers, were discarded from occupancy modeling due to low detection rates. However, species eliminated from occupancy modeling 
resulting from wide $\psi$ confidence intervals, but that presented $p>0.1$ and an appropriate associated confidence interval, were considered when determining the most influential covariates on species detectability. We created occupancy models for 10 native bird species (Table 2; Fig. 2) and 4 exotic bird species (Table 2). Covariates influencing detectability were considered for 14 native bird species (the 10 native species in Table 2 and Anairetes parulus, Curaeus curaeus, Troglodytes aedon, and Zenaida auriculata, which displayed appropriate $p$ and associated confidence intervals, but inflated $\Psi$ ).

\section{Declarations}

\section{Availability of materials and data}

The datasets generated and analyzed during the current study, along with the R code generated to analyze the data, are available from the corresponding author upon reasonable request.

\section{Acknowledgements}

We thank Fernanda Palavecino for her extensive help with obtaining urban matrix data and José Infante for his guidance with occupancy modeling. J.T.I. acknowledges the support from the Center of Applied Ecology and Sustainability-CAPES (ANID PIA/BASAL FB0002) and the ANID - Millennium Science Initiative - Center for the Socioeconomic Impact of Environmental Policies, CESIEP Code NCS13_004. This research was funded by the graduate office of the School of Agriculture and Forestry of the Pontifical Catholic University of Chile.

\section{Author contributions}

All authors contributed to the study conception and design. Material preparation, data collection and analysis were performed by CA and JDAE. The first draft of the manuscript was written by CA and JTI, JDAE, $\mathrm{CH}$, and $\mathrm{CB}$ participated in the review and editing of the manuscript. Project supervision was performed by CB. All authors read and approved the final manuscript.

\section{Funding}

This study was funded by the graduate office of the School of Agriculture and Forestry of the Pontifical Catholic University of Chile.

\section{Competing interests}

The authors declare no competing interests.

\section{References}

1. Amaya-Espinel, J.D., Hostetler M., Henríquez C. \& Bonacic, C. The influence of building abundance on Neotropical bird communities found in small urban parks. Landscape and Urban Planning. 
190(May), 103578. https://doi.org/10.1016/j.landurbplan.2019.05.009 (2019).

2. Asensio, C., Pavón, I. \& de Arcas, G. Changes in noise levels in the city of Madrid during COVID-19 lockdown in 2020. The Journal of the Acoustical Society of America.

https://doi.org/10.1121/10.0002008 (2020).

3. Bambach, N., Meza, F.J., Gilabert, H. \& Miranda, M. Impacts of climate change on the distribution of species and communities in the Chilean Mediterranean ecosystem. Regional Environmental Change. https://doi.org/10.1007/s10113-013-0425-7 (2013).

4. Basu, B., Murphy, E., Molter, A., Sarkar Basu, A., Sannigrahi, S., Belmonte, M. \& Pilla, F. Investigating changes in noise pollution due to the COVID-19 lockdown: The case of Dublin, Ireland. Sustainable Cities and Society. https://doi.org/10.1016/j.scs.2020.102597 (2021).

5. Blair, R.B. Land use and avian species diversity along an urban gradient. Ecological Applications. https://doi.org/10.2307/2269387 (1996).

6. Cardoso, G.C. \& Atwell, J.W. On the relation between loudness and the increased song frequency of urban birds. Animal Behaviour. https://doi.org/10.1016/j.anbehav.2011.07.018 (2011).

7. Carral-Murrieta, C.O., García-Arroyo, M., Marín-Gómez, O.H., Sosa-López, J.R., and MacGregor-Fors, I. Noisy environments: untangling the role of anthropogenic noise on bird species richness in a Neotropical city. Avian Research 11. BioMed Central: 32. https://doi.org/10.1186/s40657-020-002185 (2020).

8. Daude, C., Fajardo, G., Brassiolo, P., Estrada, R., Goytia, C., Sanguinetti, P., ... Vargas, J. RED 2017. Crecimiento urbano y acceso a oportunidades: un desafío para América Latina. Bogotá: CAF. http://scioteca.caf.com/handle/123456789/1090_(2017).

9. Derryberry, E.P., Phillips, J.N., Derryberry, G.E., Blum, M.J. \& Luther, D. Singing in a silent spring: Birds respond to a half-century soundscape reversion during the COVID-19 shutdown. Science. https://doi.org/10.1126/science.abd5777 (2020).

10. Fischer, J.D., Schneider, S.C., Ahlers, A.A. \& Miller, J.R. Categorizing wildlife responses to urbanization and conservation implications of terminology. Conservation Biology. https://doi.org/10.1111/cobi.12451 (2015).

11. Fiske, I.J. \& Chandler, R.B. Unmarked: An R package for fitting hierarchical models of wildlife occurrence and abundance. Journal of Statistical Software. https://doi.org/10.18637/jss.v043.i10 (2011).

12. Francis, C.D., Ortega, C.P. \& Cruz, A. Noise pollution changes avian communities and species interactions. Current biology. 19(16): 1415-1419 (2009).

13. Gill, S.A., Job, J.R., Myers, K., Naghshineh, K. \& Vonhof, M.J. Toward a broader characterization of anthropogenic noise and its effects on wildlife. Behavioral Ecology. https://doi.org/10.1093/beheco/aru219 (2015).

14. González Oreja, J.A., Bonache Regidor, C., Buzo Franco, D., De La Fuente Díaz Ordaz, A.A. \& Hernández Satín, L. Caracterización ecológica de la avifauna de los parques urbanos de la Ciudad de Puebla (México). Ardeola. 54:53-67 (2007). 
15. González-Oreja, J.A., De La Fuente-Díaz-Ordaz, A.A., Hernández-Santín, L., Bonache-Regidor, C. \& Buzo-Franco, D. Can human disturbance promote nestedness? Songbirds and noise in urban parks as a case study. Landscape and Urban Planning. https://doi.org/10.1016/j.landurbplan.2011.09.001 (2012).

16. Ikin, K., Beaty, R.M., Lindenmayer, D.B., Knight, E., Fischer, J. \& Manning, A.D. Pocket parks in a compact city: How do birds respond to increasing residential abundance? Landscape Ecology. https://doi.org/10.1007/s10980-012-9811-7 (2013).

17. Instituto Nacional de Estadísticas (INE). Estimaciones y proyecciones de la población de Chile 20022035 Regiones y áreas urbano-rural. https://www.ine.cl/. Accessed November 2020 (2019).

18. Isaksson, C. Impact of Urbanization on Birds. 235-257 in Tietze D, editors. Bird Species. Fascinating Life Sciences. Springer, Cham. https://doi.org/10.1007/978-3-319-91689-7_13 (2018).

19. Jerem, P. \& Mathews, F. Trends and knowledge gaps in field research investigating effects of anthropogenic noise. Conservation Biology. https://doi.org/10.1111/cobi.13510 (2020).

20. Luo, J., Siemers, B.M. \& Koselj, K. How anthropogenic noise affects foraging. Global Change Biology. https://doi.org/10.1111/gcb.12997 (2015).

21. McGregor, P.K. \& Dabelsteen, T. Communication networks. 409-425 in Kroodsma, D.E., Miller, E.H., editors. Ecology and Evolution of Acoustic Communication in Birds (Cornell University Press: Ithaca, NY, USA, 1997).

22. McKinney, M.L. Urbanization, biodiversity, and conservation. BioScience. https://doi.org/10.1641/0006-3568(2002)052[0883:UBAC]2.0.C0;2 (2002).

23. Mendes, S., Colino-Rabanal, V.J. \& Peris, S.J. Bird song variations along an urban gradient: The case of the European blackbird (Turdus merula). Landscape and Urban Planning. https://doi.org/10.1016/j.landurbplan.2010.08.013 (2011).

24. Mockford, E.J. \& Marshall, R.C. Effects of urban noise on song and response behaviour in great tits. Proceedings of the Royal Society B: Biological Sciences. https://doi.org/10.1098/rspb.2009.0586 (2009).

25. Nemeth, E. \& Brumm, H. Birds and Anthropogenic Noise: Are Urban Songs Adaptive? The American Naturalist. https://doi.org/10.1086/656275 (2010).

26. Nemeth, E., Pieretti, N., Zollinger, S.A., Geberzahn, N., Partecke, J., Mirand, A.C. \& Brumm, H. Bird song and anthropogenic noise: Vocal constraints may explain why birds sing higher-frequency songs in cities. Proceedings of the Royal Society B: Biological Sciences. https://doi.org/10.1098/rspb.2012.2798 (2013).

27. Ortega, C.P. Chapter 2: Effects of noise pollution on birds: A brief review of our knowledge. Ornithological monographs. 74(1): 6-22. (2012).

28. Perillo, A., Mazzoni, L.G., Passos, L.F., Goulart, V.D.L.R., Duca, C. \& Young, R.J. Anthropogenic noise reduces bird species richness and diversity in urban parks. Ibis. https://doi.org/10.1111/ibi.12481 (2017). 
29. Pijanowski, B.C., Villanueva-Rivera, L.J., Dumyahn, S.L., Farina, A., Krause, B.L., Napoletano, B.M., ... Pieretti, N. Soundscape Ecology: The Science of Sound in the Landscape. BioScience. https://doi.org/10.1525/bio.2011.61.3.6 (2011).

30. Proppe, D.S., Sturdy, C.B. \& St. Clair, C.C. Anthropogenic noise decreases urban songbird diversity and may contribute to homogenization. Global Change Biology. https://doi.org/10.1111/gcb.12098 (2013).

31. Sarricolea, P., Herrera-Ossandon, M. \& Meseguer-Ruiz, Ó. Climatic regionalisation of continental Chile. Journal of Maps. https://doi.org/10.1080/17445647.2016.1259592 (2017).

32. Slabbekoorn, H. Songs of the city: Noise-dependent spectral plasticity in the acoustic phenotype of urban birds. Animal Behaviour. https://doi.org/10.1016/j.anbehav.2013.01.021 (2013).

33. Slabbekoorn, H. \& Peet, M. Birds sing at a higher pitch in urban noise. Nature. https://doi.org/10.1038/424267a (2003).

34. Sol, D., Lapiedra, O. \& González-Lagos, C. Behavioural adjustments for a life in the city. Animal Behaviour. https://doi.org/10.1016/j.anbehav.2013.01.023 (2013).

35. United Nations, DESA (Department of Economic and Social Affairs), Population Division. World Urbanization Prospects: The 2018 Revision (ST/ESA/SER.A/420). New York: United Nations. https://population.un.org/wup/Publications/Files/WUP2018-Report.pdf. Accessed November 2020 (2019).

36. Ware, H.E., McClure, C.J.W., Carlisle, J.D. \& Barber, J.R. A phantom road experiment reveals traffic noise is an invisible source of habitat degradation. Proceedings of the National Academy of Sciences. https://doi.org/10.1073/pnas.1504710112 (2015).

37. Warren, P.S., Katti, M., Ermann, M. \& Brazel, A. Urban bioacoustics: It's not just noise. Animal Behaviour. https://doi.org/10.1016/j.anbehav.2005.07.014 (2006).

\section{Figures}


(a)

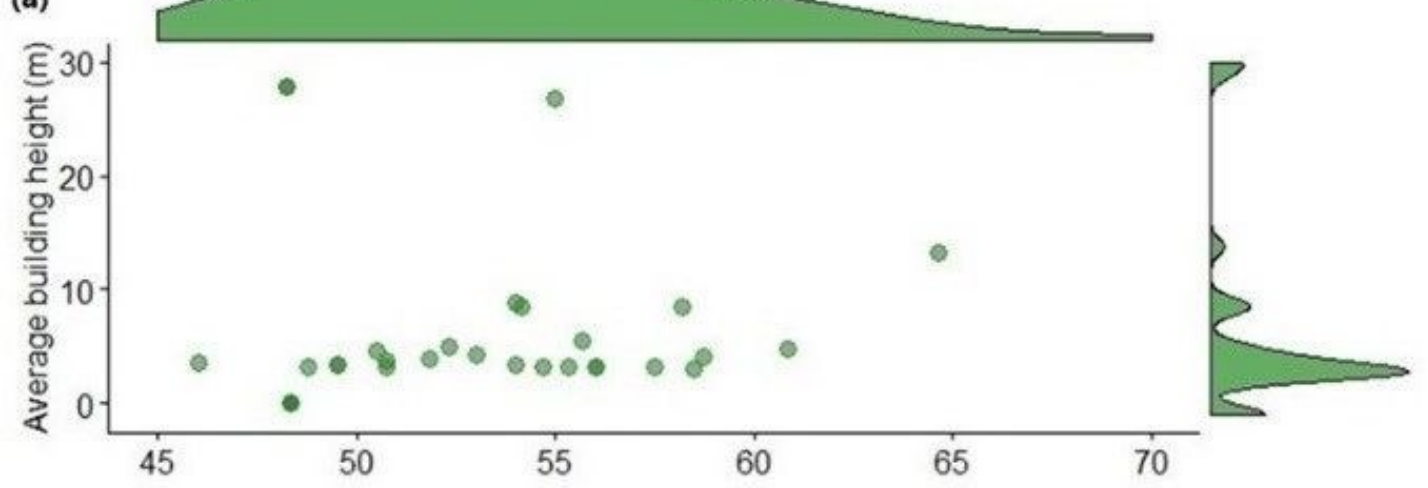

Utilizers S. sephaniodes

(b)
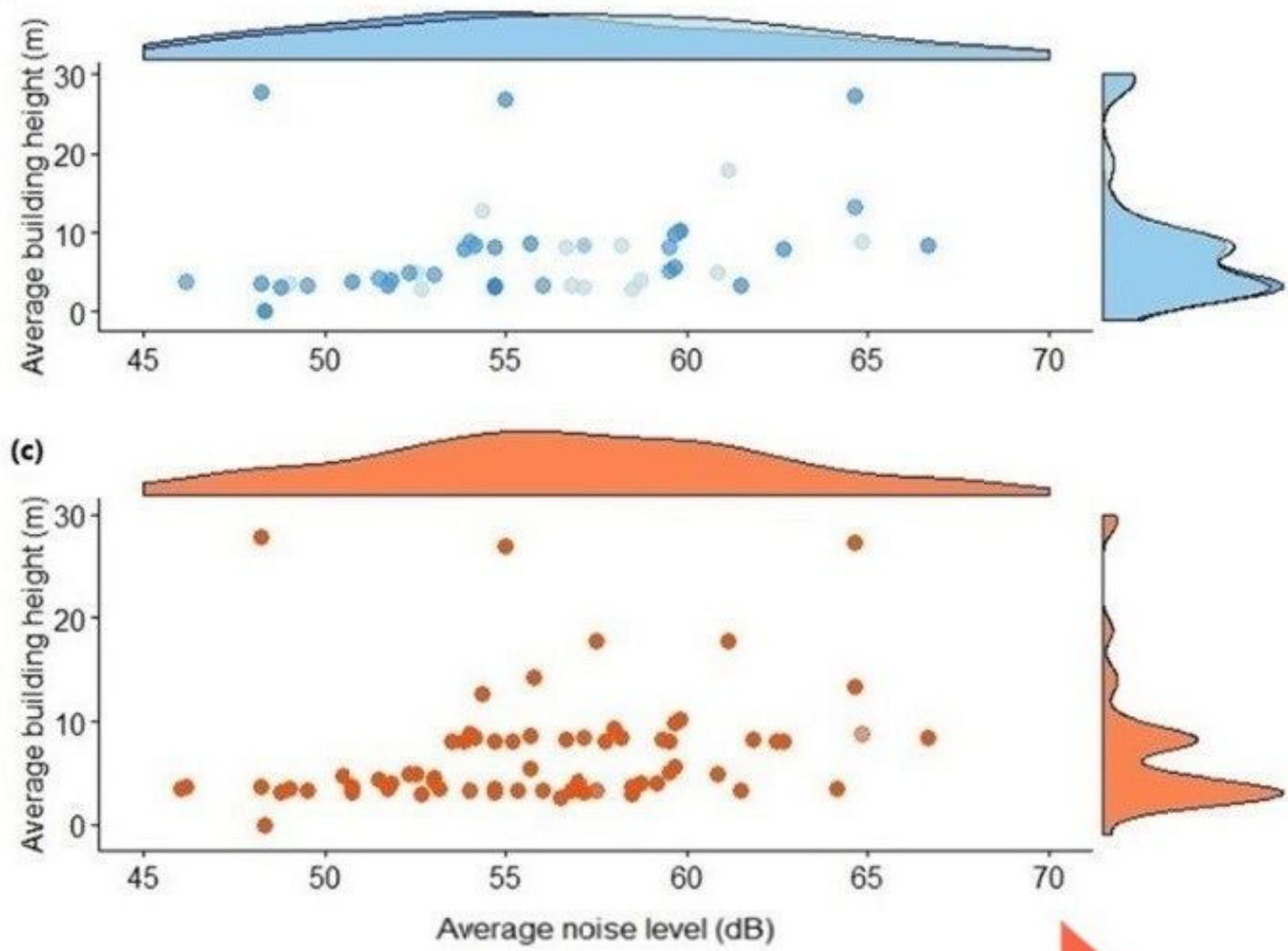

dB

50

55

60

65

70

\section{Figure 1}

Average noise levels in green spaces and average building height surrounding green spaces at which native bird species occur: a) urban avoiders, b) urban utilizers, with Sephanoides sephaniodes considered separately, and c) urban dwellers. Marginal density plots indicate the distribution of native birds at varying average noise levels in green spaces (top) and varying average building height surrounding green 
spaces (right). Decibel level comparisons (based on values established by Yale EHS) are displayed in the arrow at the bottom for reference.

(a)

\section{Leistes loyca}

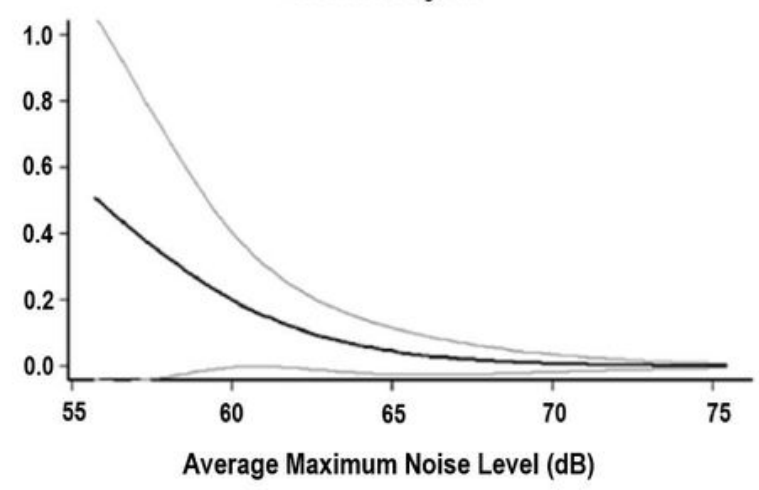

(b)
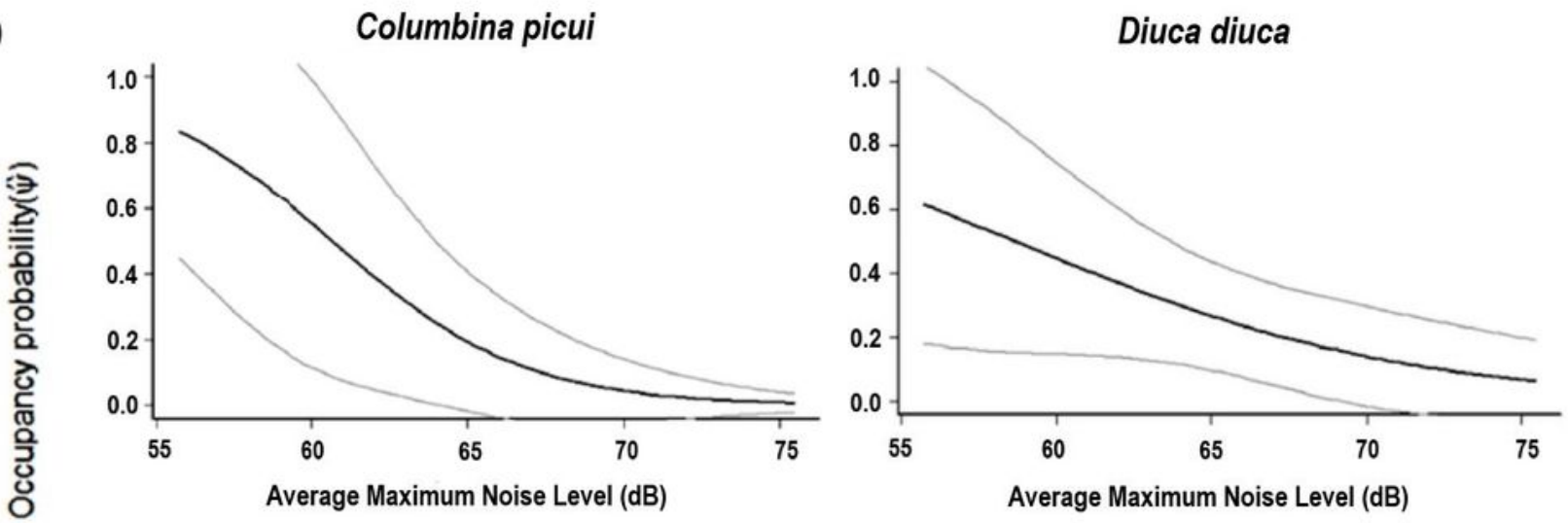

(c)

Turdus falcklandii

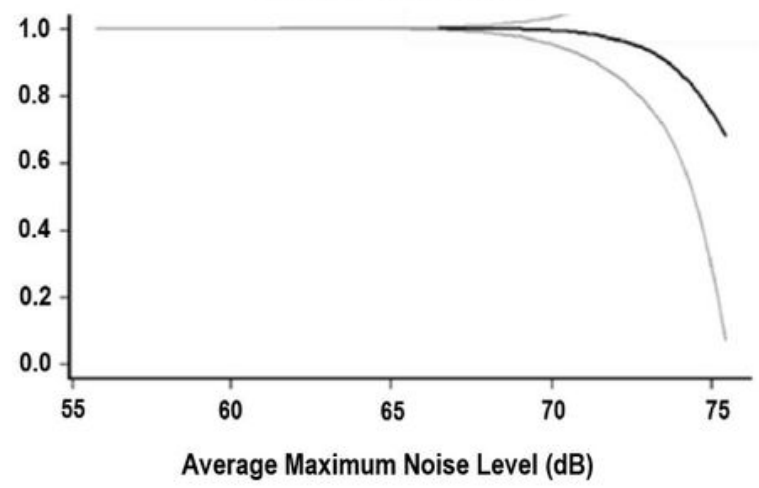

Zonotrichia capensis

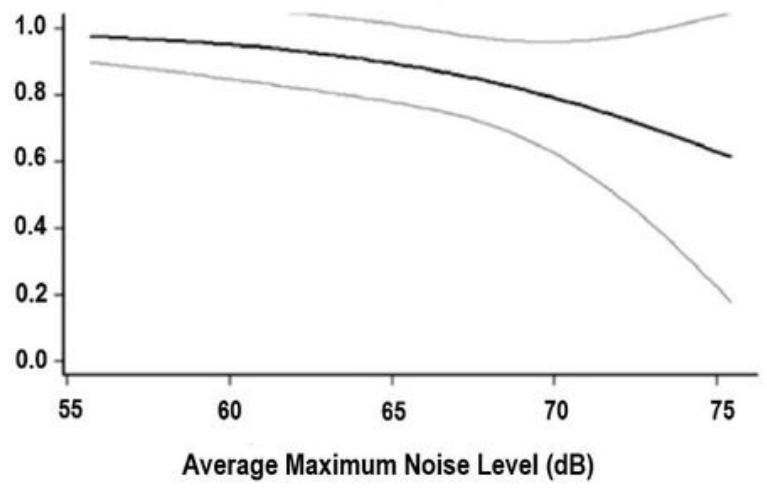

Figure 2

Occupancy probability plots for species whose occupancy was most influenced by average maximum noise levels in green spaces in Santiago, Chile: (a) native urban avoider species, (b) native urban utilizer 
species, and (c) native urban dweller species. Plots are based on the models summarized in Table 2. Gray lines represent $95 \%$ confidence intervals.

\section{Supplementary Files}

This is a list of supplementary files associated with this preprint. Click to download.

- GraphicalAbstract.pdf

- Supportinglnformation.pdf 